\title{
Kooperationen zur Digitalisierung in Lehre, Forschung und Verwaltung an den Hochschulen. Sekundärauswertung einer bundesweiten Erhebung
}

\section{Harald Gilch, Astrid Book und Klaus Wannemacher}

\section{Zusammenfassung}

Die zentrale Bedeutung von Kooperationen und Netzwerken als Strukturelement von Hochschulen für die Digitalisierung auf regionaler, nationaler und internationaler Ebene ist in ihren unterschiedlichen Dimensionen immer mehr in den Fokus gerückt. Auch die Studie „Digitalisierung der Hochschulen“ (Gilch et al. 2019), die HIS-HE 2018 im Auftrag der Expertenkommission Forschung und Innovation (EFI) durchgeführt hat, erhob Daten, die Aufschluss über Kooperationen im Bereich der Digitalisierung der Hochschulen geben. Mittels einer Sekundärauswertung dieser Daten untersucht der Beitrag, welche hochschulbezogenen Rahmenbedingungen für Kooperationen besonders förderlich sind, und analysiert, welche Spezifika die Hochschulen aufweisen, die systematisch auf Kooperationen in den Bereichen Lehre, Forschung und Verwaltung setzen. Dabei bestätigen die quantitativen Ergebnisse nicht nur frühere qualitative Schlussfolgerungen, nach denen der Reifegrad der eigenen IT-Governance einen wesentlichen Faktor für erfolgreiche Kooperationen im Bereich Digitalisierung darstellt (von der Heyde 2016). Es werden auch praktische Hinweise gegeben, welche Maßnahmen Hochschulen ergreifen sollten,

H. Gilch $(\varangle) \cdot$ A. Book $\cdot$ K. Wannemacher

HIS-Institut für Hochschulentwicklung e. V., Hannover, Deutschland

E-Mail: gilch@his-he.de

A. Book

E-Mail: book@his-he.de

K. Wannemacher

E-Mail: wannemacher@his-he.de 
wenn sie vor der Herausforderung stehen, Kooperationen noch intensiver einzugehen, um trotz der immer knapper werdenden finanziellen Ressourcen die steigenden Herausforderungen der Digitalisierung bewältigen zu können.

\section{Schlüsselwörter}

Digitalisierung $\bullet$ Hochschule $\bullet$ Kooperation $\bullet$ Verbund $\bullet$ Netzwerk $\bullet$ Lehre $\bullet$ Forschung $\bullet$ Verwaltung $\bullet$ Governance $\bullet$ Hochschulentwicklung

\section{$1 \quad$ Einleitung}

Hochschulbezogenen Kooperationen wird ein „unglaubliches Leistungspotenzial“ (Schiewer 2019, S. 18) zugeschrieben, das sowohl die Hochschulen selbst und die dort gelehrten Disziplinen als auch die Forschung und in indirekter Weise auch Studierende betrifft. Auf dem Weg über Kooperationen soll ein Mehrwert erzielt werden, der den Institutionen allein verwehrt bliebe (ebenda). Die Ausgangspunkte von Kooperationen in Gestalt eines ,handelnde[n] Zusammenwirken[s] von individuellen Akteuren [...] sind dabei sehr unterschiedlich“ (Wagner et al. 2019, S. 4). Ihnen gemein ist jedoch eine gewisse Bereitschaft, Ressourcen wie Zeit, Geld und Energie zu investieren (Schiewer 2019, S. 18). Als fördernde Faktoren für Kooperationen gelten außerdem die Kompetenz und Motivation der Mitarbeitenden (von Suchodoletz 2016, S. 186); weitere wichtige Treiber von Kooperationen sind beispielsweise die Konvergenz von Zielen und Interessen, die Bewältigung von gemeinsamen Herausforderungen oder die Förderung und Unterstützung von (regionalen) Kooperationen durch die Politik (Winde et al. 2017, S. 3).

Die zentrale Bedeutung unterschiedlicher Arten von Kooperationen und Netzwerken auf regionaler, nationaler und internationaler Ebene für die Digitalisierung der Hochschulen ist in den vergangenen Jahren zunehmend erkannt und in ihren unterschiedlichen Dimensionen näher untersucht worden. (zum Beispiel Pongratz 2015; Schünemann und Budde 2018; Wachtler et al. 2016; Wannemacher und Geidel 2016). Im Kontext der Peer-to-Peer-Beratung des Hochschulforums Digitalisierung wurde als eine der wesentlichen Strukturentscheidungen, die Hochschulen als Basis für die Weiterentwicklung der Lehre unter dem Vorzeichen der Digitalisierung treffen können, ein systematischer Ausbau von Hochschulkooperationen identifiziert: „Gerade im Kontext der Digitalisierung sind Hochschulkooperationen der Schlüssel, um den digitalen Wandel erfolgreich zu gestalten, bestehende 
Formen der Zusammenarbeit auszubauen und neue Angebote zu entwickeln“ (Schünemann und Budde 2018, S. 23).

Dieser hohen Bedeutung von Kooperationen für die Digitalisierung speziell im Bereich Lehre tragen auch die Rechenzentren als die zentralen IT-Dienstleister in den Hochschulen Rechnung. Dem Erfordernis, in den Hochschulen ein immer größeres Portfolio an digitalen Diensten mit gleichbleibend hoher Qualität anzubieten und dabei zusätzlich in Konkurrenz zu großen internationalen IT-Unternehmen aus der freien Wirtschaft zu stehen, „können die Rechenzentren nur begegnen, indem sie sich zusammentun, sich spezialisieren oder Dienste hochschulübergreifend anbieten" (von Suchodoletz et al. 2016, S. VI). HIS-HE hat in 2018 im Rahmen der Studie „Digitalisierung der Hochschulen“ (Gilch et al. 2019) im Auftrag der Expertenkommission Forschung und Innovation (EFI) eine teilstandardisierte Vollerhebung unter den Leitungen aller deutschen Hochschulen durchgeführt, wobei 119 Antworten von Hochschulen unterschiedlichen Typs und unterschiedlicher Größe aus allen Bundesländern vorliegen. Auch wenn sich das hohe Potenzial von Kooperationen im Rahmen dieser Studie wieder gezeigt hat, kommen die Studie und daraus abgeleitet auch die EFI in ihrem Jahresgutachten 2019 zu dem Schluss, dass ebendieses Potenzial bei der Digitalisierung von Hochschulen noch nicht ausgeschöpft sei (EFI 2019, S. 100). Insofern hat HIS-HE die quantitativen Daten der Studie im Rahmen einer Sekundärauswertung noch einmal mithilfe deskriptiver Analysemethoden betrachtet, um zu überprüfen, inwieweit die Daten weitere Erkenntnisse über den Einfluss von Kooperationen auf eine erfolgreiche Digitalisierung wie auch über Rahmenbedingungen für erfolgreiche Kooperationen liefern können.

\section{Ergebnisse der Studie zur Digitalisierung der Hochschulen}

Im Hinblick auf die Bedeutung und Reichweite von Kooperationen bei der Digitalisierung der Hochschulen ergab die Studie im Jahr 2018 unterschiedliche Befunde. Die Hochschulen und Fachhochschulen schließen in allen Bereichen Verbünde und Kooperationen ab. Die wichtigste Rolle in diesem Zusammenhang spielen aus Sicht der befragten Hochschulleitungen Kooperationen zur Digitalisierung des Lehrens und Lernens $(72,1 \%)$ und der Digitalisierung der Infrastruktur (67,3\%). Kooperationen im Bereich der digitalen Verwaltung, der digitalen Forschung und der Gesamtinstitution Hochschule treten hingegen weniger häufig auf. Auffällig ist in diesem Zusammenhang auch, dass Universitäten 


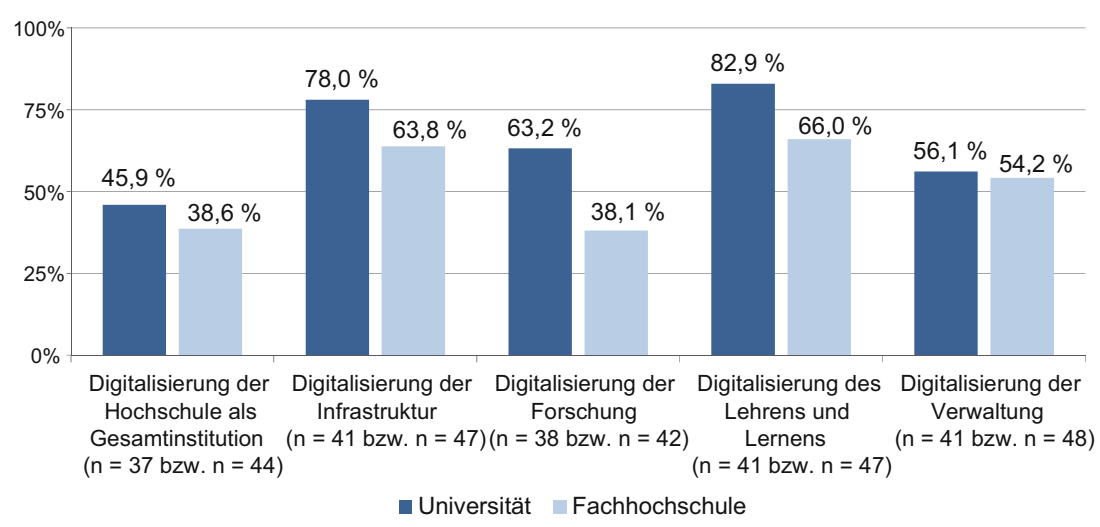

Abb. 1 Verbünde und Kooperationen zur Digitalisierung nach Hochschultyp: „Existieren Verbünde oder Kooperationen mit anderen Hochschulen, die das Ziel der Digitalisierung in den folgenden Bereichen verfolgen?" Anteil der Zustimmungen nach Hochschultypen. (Gilch et al. 2019, S. 129)

deutlich häufiger Kooperationen eingehen und Verbünde gründen als Fachhochschulen beziehungsweise Hochschulen für angewandte Wissenschaften es tun (vgl. Abb. 1). Bezüglich der Reichweite der Kooperationen und Verbünde dominiert über alle Bereiche die Zusammenarbeit auf der Ebene des Bundeslandes (vgl. zur Lehre: Wannemacher et al. 2016, S. 32-35). Vor allem im Bereich Infrastruktur spielen aber auch Kooperationen zwischen einzelnen Hochschulen sowie auf regionaler Ebene schon seit Langem eine wichtige Rolle.

In diesem Zusammenhang seien zum Beispiel das Leibniz-Rechenzentrum der Bayerischen Akademie der Wissenschaften für die Münchner Universitäten LMU und TUM sowie die Akademie der Wissenschaften, die Gesellschaft für wissenschaftliche Datenverarbeitung mbH Göttingen (GWDG) als gemeinsame Einrichtung der Universität Göttingen und der Max-Planck-Gesellschaft sowie das Regionale Hochschulrechenzentrum Kaiserslautern (RHRK) als gemeinsame Einrichtung der Rechenzentrumsallianz Rheinland-Pfalz (RARP) mit allen rheinland-pfälzischen Hochschulen genannt. $\mathrm{Zu}$ bundesweiten Zusammenschlüssen zählen beispielsweise gemeinsame Einrichtungen wie HIS eG oder DFN e. V., die als Genossenschaft bzw. Verein organisiert sind und Campus-ManagementSysteme entwickeln beziehungsweise ein Kommunikationsnetz für Wissenschaft und Forschung betreiben. 
Ein anderes wichtiges Ergebnis der Studie betrifft die Strategie und Governance der Digitalisierung. Die Hochschulen haben in den letzten Jahren vermehrt Strukturen für die IT-Governance (in Form von Zuständigkeiten bei der Hochschulleitung bzw. als CIO oder CIO-Gremium) eingerichtet und es zeigte sich - wenn auch angesichts der niedrigen Fallzahlen nur mit geringer statistischer Relevanz - ein positiver Effekt, insofern sich mit der Einrichtung eines CIO auch der Stellenwert, der Stand und das Erarbeiten einer Digitalisierungsstrategie erhöht.

Die IT-Governance und die Kooperationsbestrebungen von Hochschulen zu kontextualisieren und daraus Aussagen hinsichtlich Kooperationen und Governance abzuleiten, ist Gegenstand der nachfolgenden Abschnitte.

\section{$3 \quad$ Ergebnisse der Sekundärauswertung (quantitativ)}

Die Sekundärauswertung der Daten aus der Studie zeigt eine Reihe von Tendenzen, die vielleicht nicht ganz überraschend sind, die aber in dieser Form nun zum ersten Mal auch quantitativ bestätigt werden konnten:

1. Hochschulen, die Kooperationen zur Digitalisierung verfolgen, geben einen vergleichsweise höheren Stellenwert von Digitalisierung an. Während der Anteil der Hochschulen mit hohem Stellenwert im Bereich des Lehrens und Lernens von 73,3\% (ohne Kooperationen) auf 77,9\% (mit Kooperationen) nur moderat ansteigt, ist der Unterschied im Bereich Verwaltung deutlich höher. Hier geben 79,0 \% der Hochschulen mit Kooperationen an, der Digitalisierung im Bereich Verwaltung einen hohen Stellenwert beizumessen, während dies nur für 62,2\% der Hochschulen ohne Kooperationen gilt.

2. An Hochschulen, die in höherem Maße Kooperationen zur Digitalisierung verfolgen, befindet sich gleichzeitig überproportional häufig ein $\mathrm{CIO}$ oder ein CIO-Gremium (vgl. Abb. 2). Abgesehen vom Bereich Verwaltung liegt der Anteil der Hochschulen mit CIO bei Kooperationen um mehr als $10 \%$ höher als ohne Kooperationen. So verfügen beispielsweise 68,4 \% der Hochschulen, die angeben, eine Digitalisierungskooperation als Gesamtinstitution eingegangen zu sein, über einen CIO. Bei Hochschulen, die keine derartige Kooperation angeben, fällt dieser Wert mit 55,4\% deutlich geringer aus.

3. An Hochschulen, die in höherem Maße Kooperationen zur Digitalisierung verfolgen, existieren mit 78,6\% im Vergleich zu Hochschulen, die nicht auf Kooperationen abzielen $(68,9 \%)$, auch häufiger zentrale Organisationseinheiten zur Unterstützung der Lehrenden im Bereich Digitalisierung des 
Lehrens und Lernens. Explizit zwei Hochschulen (von 117) weisen zusätzlich darauf hin, eine solche Organisationseinheit extern (also im Rahmen einer Kooperation oder eines Verbundes) zu betreiben.

4. 74,0 \% der Hochschulen, die eine Kooperation zur Digitalisierung der Lehre und des Lernens verfolgen, geben an, ein schriftliches Konzept zur Digitalisierung des Lehrens und Lernens derzeit auszuarbeiten oder ausgearbeitet zu haben (ohne Kooperation $=60,7 \%$ ) (vgl. Abb. 3). 64,9\% der Hochschulen kooperieren im Bereich der Digitalisierung der Verwaltung und verfügen über eine schriftliche Strategie dazu (ohne Kooperation $=61,9 \%$ ).

5. An Hochschulen mit Kooperationen zur Digitalisierung ist die Einstellung der Mitarbeiter*innen gegenüber der Digitalisierung im Durchschnitt positiver. Besonders deutlich ist dieser Unterschied, wenn die Einstellungen der Mitarbeiter*innen aus Technik und Verwaltung betrachtet wird: An Hochschulen, die Kooperationen zur Digitalisierung der Verwaltung eingegangen sind, wird deren Einstellung zur Digitalisierung durchschnittlich um ca. 0,5 Punkte zustimmender eingeschätzt als an Hochschulen, die keine Kooperation in diesem Bereich verfolgen (3,7 vs. 3,2 auf einer Skala von $1=$ sehr gering bis 5 $=$ sehr hoch).

Auch wenn, wie erwähnt, jede Aussage für sich angesichts der Fallzahlen immer nur begrenzt statistisch belastbar ist, belegt die Gesamtheit der Einzelergebnisse doch eindeutig: Kooperationen im Bereich Digitalisierung werden an Hochschulen eher dann eingegangen, wenn der Digitalisierung ein hoher Stellenwert zugesprochen wird, die Hochschulen selbst über eine ausgereifte interne Governance verfügen, die Hochschulleitung in die Digitalisierung strategisch eingebunden ist und Digitalisierung auch strukturell über einen CIO oder ein Äquivalent in der Hochschule als Ganzes verankert ist. Gleichzeitig zeigt sich ein positiver Zusammenhang zwischen der Bereitschaft der Hochschule, Kooperationen einzugehen, und der Einstellung der Mitarbeitenden gegenüber der Digitalisierung. Wagner et al. bestätigen, dass ,fehlendes Vertrauen auf Individualebene [...] den Aufbau funktionierender Formen der Zusammenarbeit auf Ebene des Verbundes [erschwert]“ (Wagner et al. 2019, S. 6).

Inwiefern diese Aussagen tatsächlich kausal bedingt sind, können die quantitativen Daten in dieser Form nicht belegen. Die Analyse qualitativer Aussagen der in der Studie untersuchten Fallbeispiele sowie die Untersuchung der Sekundärliteratur, wie sie im folgenden Abschnitt vorgenommen wird, liefert jedoch entsprechende Hinweise. 


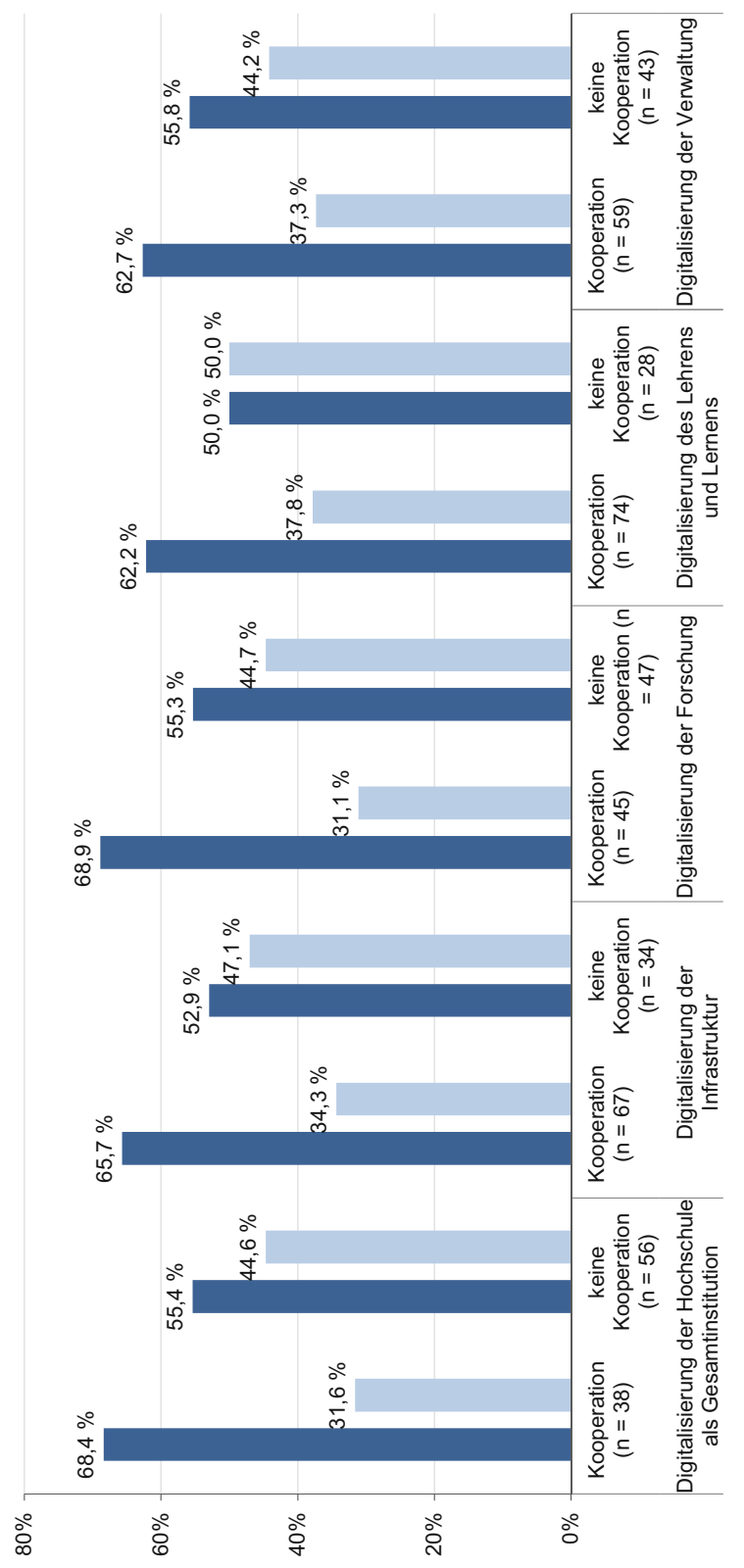




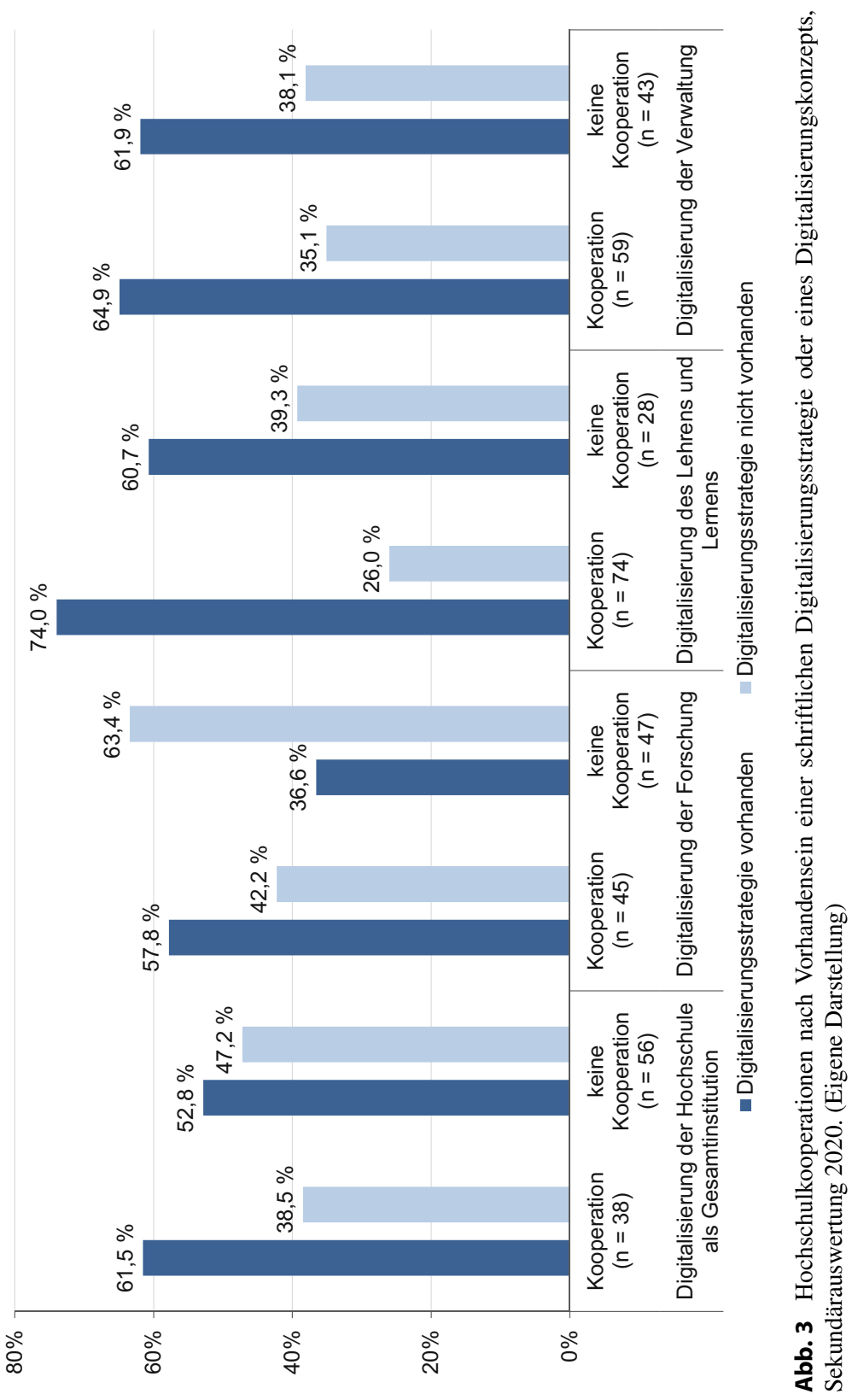




\section{$4 \quad$ Ergebnisse der Sekundärauswertung (qualitativ)}

Für die Studie hat HIS-HE zusätzlich zur quantitativen Erhebung im Rahmen einer Untersuchung von zehn Fallbeispielen auch qualitative Daten erhoben. Diese beruhen auf Interviews mit verschiedenen Akteur*innen (Präsident*innen, Kanzler*innen, CIOs, Lehrende, Studierende), die im Sommer 2018 unter anderem zu ihrer Haltung bezüglich Kooperationen befragt worden sind und im Folgenden beispielhaft zitiert werden. Insbesondere Hochschulleitungen und CIOs zeigen für Kooperationen grundsätzlich positive Grundhaltungen und verbinden gute Erfahrungen damit. Wichtig sei dabei für diese generell, ,dass gemeinsame Ziele der Kooperationspartner*innen vorhanden sind" und dass sich Kooperationen ,nicht im ,Wünsch dir was“ beziehungsweise Aufnehmen der individuellen Anforderungen aller Hochschulen verlieren“. Gleichzeitig böten Kooperationen viel Potenzial, da zum Beispiel ,der Standard-IT-Betrieb (zum Beispiel E-Mail, E-Learning) nicht von jeder Hochschule selbst erbracht werden muss“. Interne Treiber sind dabei auch die Rechenzentrumsleitungen, die sich nicht nur seit Langem mit der Thematik Kooperation beschäftigen, sondern auch die Standardisierung der IT-Services vorantreiben - sowohl innerhalb der Hochschulen, wenn es darum geht, dezentral gewachsene IT-Strukturen schrittweise zu vereinheitlichen, als auch in der Zusammenarbeit mit anderen Hochschulen und externen Dienstleistenden. Das ,Ziel der Kooperationen ist nicht die Einsparung von Mitteln, sondern die professionellere Leistungserbringung“. Als weitere „Grundvoraussetzung der Kooperationen“ werden „Personen mit entsprechender Berechtigung und Initiative sowie die Offenheit der Institution“ gesehen. „Auf Leitungsebene funktioniert die Zusammenarbeit gut. Auf der Ebene der einzelnen Mitarbeiter*innen muss die Zusammenarbeit noch verbessert werden." Der quantitative Zusammenhang zwischen positiver Einstellung als Basis für mehr Kooperation wird damit qualitativ bestätigt.

Trotz dieser grundsätzlichen Zustimmung zu Kooperationen gibt es in der Praxis jedoch auch viele Bedenken und Hemmnisse, die von den Befragten benannt werden. Dazu gehören unter anderem die Erfordernisse der Standardisierung und die Notwendigkeit, sich auf ein gemeinsames Vorgehen zu einigen, obwohl sich viele Hochschulen doch ,als so besonders wahrnehmen“. Zudem besteht die Befürchtung: „Mit Kooperationen gehen wir auch Verpflichtungen ein. Daher arbeiten wir lieber in eigener Regie“ (Gilch et al. 2019, S. 133). Diese Verpflichtungen beginnen bereits bei der Einhaltung der ,Vielzahl an möglichen Organisations- und Rechtsnormen“ (Wagner et al. 2019, S. 7), die den Manager*innen der Kooperationen ein breites Fachwissen abverlangen. In diesem Zusammenhang wird in der vorliegenden qualitativen Untersuchung immer 
wieder die Umsatzsteuergesetzgebung als hemmend bezeichnet, da nach $\S 2 \mathrm{~b}$ UStG Kooperationen zwischen juristischen Personen des öffentlichen Rechts nur dann umsatzsteuerbefreit sind, wenn diese einem gemeinsamen spezifischen öffentlichen Interesse dienen. Dieses muss jedoch gegenüber den Finanzbehörden nachgewiesen werden, wobei zusätzlich keine schädlichen Wettbewerbsverzerrungen vorliegen dürfen. Wie in Gilch et al. (2019, S. 134 ff.) ausgeführt wird, liegen die Voraussetzungen nach $\S 2 \mathrm{~b}$ UStG für Kooperationen im Bereich Digitalisierung grundsätzlich dann vor, wenn entsprechende gesetzliche Bestimmungen für die gemeinsame Aufgabenwahrnehmung von staatlichen Hochschulen und Forschungseinrichtungen in den Landeshochschulgesetzen vorhanden sind - dies ist jedoch bisher nur in wenigen Bundesländern der Fall (zum Beispiel in Bremen: $\S 13$ BremHG, in Nordrhein-Westfalen: $\S 77$ Abs. 2 und 4 HG NRW oder in Thüringen: § 37 Abs. 4 ThürHG).

Wir stellen also fest: Kooperationen im Bereich Digitalisierung werden grundsätzlich als sinnvoll erachtet, in der Praxis überwiegen jedoch häufig Bedenken oder es werden hinderliche Rahmenbedingungen als Gründe genannt, die Kooperationen entgegenstehen. Hier sind also strategisches Handeln und Entscheidungen auf oberster Ebene notwendig, wenn diese Bedenken überwunden werden sollen. Insofern stellen wir eine Parallelität hinsichtlich interner Erfolgsfaktoren für die erfolgreiche Digitalisierung fest, denn auch hierfür ist die Notwendigkeit der strategischen Verankerung von Digitalisierung in der Hochschulleitung und die Bedeutung eines CIO oder CIO-Gremiums unbestritten (Gilch et al. 2019, S. 99). Die Schlussfolgerung von von der Heyde (2016), dass erfolgreiche Kooperationen in vielen Fällen letztlich mit einem hohen Reifegrad von Governance und Entscheidungsstrukturen zusammenhängen, wird hier bekräftigt und auch von den quantitativen Daten der Studie gestützt.

Damit stellt sich die Frage: Ist dieser Zusammenhang aus Ursache und Wirkung monodirektional oder lassen sich auch Indizien dafür finden, dass gute Kooperationen förderlich für interne Governance und positive Einstellungen der Beschäftigten gegenüber der Digitalisierung sein können? Sowohl die im Rahmen der Studie durchgeführten Gespräche und Interviews als auch die Analyse der entsprechenden Literatur lassen hierfür keine Anhaltspunkte erkennen. Der Wirkungszusammenhang von guter Governance und positiver Grundeinstellung hin zu mehr Kooperationen und damit auch zur Überwindung der Hemmnisse von Kooperationen scheint letztlich plausibel. 


\section{$5 \quad$ Fazit und Ausblick}

Wie sich im Rahmen der Sekundärauswertung sowohl der quantitativen als auch der qualitativen Daten der Studie zur Digitalisierung der Hochschulen auf Grundlage der Angaben der befragten Hochschulleitungen zeigt, ist die Frage, ob Hochschulen eine Kooperation zur Digitalisierung eingehen und beibehalten, nicht nur von gemeinsamen Zielen oder Herausforderungen sowie guten externen Rahmenbedingungen (zum Beispiel Initiativen der Politik, Gilch et al. 2019, S. 153) abhängig, sondern anscheinend auch eng mit der internen IT-Governance und IT-Strategie verknüpft. Die Hochschulleitung muss Kooperationen unterstützen und Hemmnisse überwinden wollen, damit die operativen Bereiche diese eingehen und die notwendigen Voraussetzungen schaffen können. Gleichzeitig ist Kooperation aber immer auch eine Frage der digitalen Voraussetzungen und der Offenheit an der Hochschule, wie auch Wagner et al. hervorheben: „Allerdings können langjährige Beziehungen wiederum innovationshemmend wirken und in Verbünden und Kooperationen zu Silodenken führen (zu starre Fixierung auf die eigene Abteilung beziehungsweise das bisher vertraute Team), wodurch es zu einem ineffektiven Nebeneinander statt zu einem produktiven Miteinander kommt" (Wagner et al. 2019, S. 6).

Hochschulen, die vergleichsweise wenig auf digitale Prozesse umgestellt haben und an denen sich digitale Lösungen und Medien bei den Mitarbeitenden noch zu wenig etabliert haben, werden weniger oft Kooperationen eingehen. Gleichzeitig sind Kooperationen zur Digitalisierung durchaus stark vom gegenseitigen Lernen geprägt und helfen den Hochschulen dabei, ihre eigenen IT-Strukturen und IT-Systeme weiterzuentwickeln. Dabei können Kooperationen selbstverständlich keine Lösung für sämtliche Problemsituationen im digitalen Kontext der Hochschule beisteuern, weshalb gemeinsame Ziele vor Beginn einer Kooperation deutlich herausgearbeitet werden müssen (vgl. Wagner et al. 2019, S. 4). Der Aufbau einer guten Governance für die Kooperation, die Sicherstellung der Finanzierung und die Freiwilligkeit einer Beteiligung sind wichtige Grundvoraussetzungen, damit Kooperationen auch längerfristig funktionieren und für alle Beteiligten erfolgreich verlaufen (von Suchodoletz et al. 2016; Schiewer 2019, S. 20).

Während bisher Kooperationen in der Digitalisierung von den Hochschulen überwiegend als möglich, sinnvoll und hilfreich angesehen wurden, führen weitere Faktoren gegenwärtig sogar zur Notwendigkeit von Kooperationen. Es wird damit für die Hochschulen zukünftig noch wichtiger zu kooperieren: 
1. An immer mehr Hochschulen stellt sich die Ressourcenfrage. Neue ITTechnologien erfordern zunehmend auch mehr IT-Personal mit immer spezielleren Kenntnissen, wobei es jedoch für Hochschulen nicht nur schwierig ist, IT-Personal zu rekrutieren, sondern auch das Personaltableau kaum Aufstockungen erlaubt. Insofern werden unter CIOs Outsourcings zunehmend beliebter (Zastrocky 2019), wobei Kooperationen mit anderen Hochschulen hierfür eine Option eröffnen, ohne sich direkt in den Markt zu begeben. Dies kann insbesondere für kleinere Hochschulen und speziell Hochschulen für angewandte Wissenschaften zukünftig eine Option sein, zumal sie, wie sich in der Studie gezeigt hat, bisher noch weniger Kooperationen eingehen als Universitäten.

2. Die Förderung und der Ausbau von Kooperationen der Hochschulen im Bereich der Digitalisierung wird von der Politik immer mehr unterstützt und vorangetrieben. So weisen Landeshochschulentwicklungspläne wie zum Beispiel in Nordrhein-Westfalen (Landesregierung NRW 2016, S. 52) schon länger auf die Notwendigkeit von Kooperationen hin und aktuelle Regierungsprogramme wie etwa in Hessen konkretisieren die Notwendigkeit hochschulübergreifender Lösungen und länderübergreifender Initiativen (Koalitionsvertrag Hessen 2018, S. 188). Es bilden sich immer mehr Netzwerke zur Digitalisierung (zum Beispiel IuK Baden-Württemberg, Digitale Hochschule NRW), die nicht nur als Plattformen für die gemeinsame virtuelle Lehre dienen, sondern die gesamte Bandbreite der Digitalisierung bearbeiten und die Hochschulen dabei unterstützen, auch das oben genannte Ressourcenproblem anzugehen. Die länderbezogene Ausrichtung ist dabei wesentlich auch der Finanzierung geschuldet, werden diese Netzwerke doch mit Landesmitteln unterstützt, die letztlich den Hochschulen des Landes zugutekommen sollen.

3. Gesetzliche Vorschriften wie zum Beispiel das Onlinezugangsgesetz (OZG), das auch von den Hochschulen bis Ende 2022 umgesetzt werden soll, lassen sich ohne Kooperation und Zusammenarbeit nicht realisieren. Zielsetzung des OZG ist es, den Bürger*innen öffentliche Dienstleistungen komplett digital anzubieten, womit zum Beispiel ein intensiver digitaler Datenaustausch zwischen den Hochschulen sowie Hochschulen und weiteren Institutionen notwendig wird. Damit verbunden sind gemeinsame Standards, die derzeit geschaffen und in Zukunft möglichst gemeinsam umgesetzt werden. Außerdem sollen sich die Hochschulen wie auch andere Einrichtungen in Portalverbünden zusammenschließen und übergreifende Authentifizierungsroutinen nutzen. Auch dies wird kaum ohne eine intensive Zusammenarbeit zu bewältigen sein, wie sie zum Beispiel im Hinblick auf das deutsche Forschungsnetz schon mit dem DFN e. V. besteht. 
Die Hochschulen stehen also vermehrt vor der Herausforderung, Kooperationen eingehen zu müssen, auch wenn das Prinzip der Freiwilligkeit und die Vorabklärung der damit verbundenen Ziele keinesfalls vernachlässigt werden dürfen. Wie sich im Ergebnis der hier vorgelegten Analysen aber gezeigt hat, entstehen erfolgreiche Kooperationen im Bereich der Digitalisierung nicht einfach ,auf Wunsch“ oder ,per Definition“. Vielmehr gilt es vorab, innerhalb der Hochschulen günstige Rahmenbedingungen zu schaffen, wobei sich zeigt, dass der Aufbau einer „reifen" IT-Governance und die Verankerung von Digitalisierung in Hochschulleitung und Hochschulstrategie förderliche Faktoren dafür darstellen.

\section{Literatur}

EFI - Expertenkommission Forschung und Innovation. (2019). Gutachten zu Forschung, Innovation und technologischer Leistungsfähigkeit Deutschlands 2019. Berlin: EFI.

Gilch, H., Beise, A. S., Krempkow, R., Müller, M., Stratmann, F., \& Wannemacher, K. (2019). Digitalisierung der Hochschulen. Ergebnisse einer Schwerpunktstudie für die Expertenkommission Forschung und Innovation. Berlin: EFI.

Koalitionsvertrag zwischen CDU Hessen und BÜNDNIS 90/DIE GRÜNEN Hessen für die 20. Legislaturperiode. (2018). Aufbruch im Wandel durch Haltung, Orientierung und Zusammenhalt. https://www.hessen.de/sites/default/files/media/staatskanzlei/koalit ionsvertrag_20._wahlperiode.pdf. Zugegriffen: 23. Apr. 2020.

Landesregierung NRW. (2016). Landeshochschulentwicklungsplan Nordrhein-Westfalen (LHEP NRW). Gesetz- und Verordnungsblatt für das Land Nordrhein-Westfalen, 33 (18. November).

Pongratz, H. (Hrsg.). (2015). Neue Kooperations- und Finanzierungsmodelle in der Hochschullehre (Arbeitspapier, 13). Berlin: Hochschulforum Digitalisierung. https://doi.org/ 10.5281/zenodo.4282244.

Schiewer, H.-J. (2019). Wenn Universitäten kooperieren, können sie ein unglaubliches Leistungspotenzial entwickeln. DUZ Wissenschaft \& Management, 2019(8), 18-20.

Schünemann, I., \& Budde, J. (2018). Hochschulstrategien für die Lehre im digitalen Zeitalter: Keine Strategie wie jede andere! (Arbeitspapier, 38). Berlin: Hochschulforum Digitalisierung. https://doi.org/10.5281/zenodo.2592258.

von der Heyde, M. (2016). Replikations- und Diversifikationsmodelle für IT-Governance in Hochschulverbünden. In D. von Suchodoletz, J. C. Schulz, J. Leendertse, H. Hotzel \& M. Wimmer (Hrsg.), Kooperation von Rechenzentren - Governance und Steuerung - Organisation, Rechtsgrundlagen, Politik (S. 299-306). Berlin: De Gruyter. https://doi. org/10.1515/9783110459753-015.

von Suchodoletz, D. (2016). Zusammenarbeit gestalten - Stand in Baden-Württemberg. In D. von Suchodoletz, J. C. Schulz, J. Leendertse, H. Hotzel \& M. Wimmer (Hrsg.), Kooperation von Rechenzentren - Governance und Steuerung - Organisation, Rechtsgrundlagen, Politik (S. 171-188). Berlin: De Gruyter. https://doi.org/10.1515/9783110459753-015.

von Suchodoletz, D., Schulz J. C., Leendertse, J., Hotzel, H., \& Wimmer, M. (Hrsg.). (2016). Kooperation von Rechenzentren - Governance und Steuerung - Organisation, 
Rechtsgrundlagen, Politik. Berlin: De Gruyter. https://doi.org/10.1515/978311045975 3-015.

Wachtler, J., Ebner, M., Gröblinger, O., Kopp, M., Bratengeyer, E., Steinbacher, H.-P., Freisleben-Teutscher, C., \& Kapper, C. (Hrsg.). (2016). Digitale Medien: Zusammenarbeit in der Bildung. Medien in der Wissenschaft, 71. Münster: Waxmann.

Wagner, N., Kopp, J., Nieveler, S., \& Winde, M. (2019). Smart starten: Wie der Einstieg in Hochschulkooperation gelingt. Future Lab: Kooperationsgovernance. Diskussionspapier, 2. Essen: Stifterverband für die Deutsche Wissenschaft e. V.

Wannemacher, K., \& Geidel, J. (2016). Digitale Modelle internationaler Hochschulkooperation in der Lehre (Arbeitspapier, 22). Berlin: Hochschulforum Digitalisierung. https:// doi.org/10.5281/zenodo.4282206.

Wannemacher, K., Jungermann, I., Osterfeld, S., Scholz, J., \& v. Villiez, A. (2016). Organisation digitaler Lehre in den deutschen Hochschulen (Arbeitspapier, 21). Berlin: Hochschulforum Digitalisierung. https://doi.org/10.5281/zenodo.4282224.

Winde, M., Dauchert, A., Leusing, B., \& Meyer-Guckel, V. (2017). Durch Kooperation zum Standortprofil: Partnerschaften von Universitäten und Fachhochschulen - Strategien und Fallbeispiele. Essen: Edition Stifterverband.

Zastrocky, M. (Hrsg.). (2019). Information technology in higher education 2019. Ten years of surveys of chief information officers in Higher Ed. Broomfield, CO: Leadership Board for CIOs in Higher Education.

Open Access Dieses Kapitel wird unter der Creative Commons Namensnennung 4.0 International Lizenz (http://creativecommons.org/licenses/by/4.0/deed.de) veröffentlicht, welche die Nutzung, Vervielfältigung, Bearbeitung, Verbreitung und Wiedergabe in jeglichem Medium und Format erlaubt, sofern Sie den/die ursprünglichen Autor(en) und die Quelle ordnungsgemäß nennen, einen Link zur Creative Commons Lizenz beifügen und angeben, ob Änderungen vorgenommen wurden.

Die in diesem Kapitel enthaltenen Bilder und sonstiges Drittmaterial unterliegen ebenfalls der genannten Creative Commons Lizenz, sofern sich aus der Abbildungslegende nichts anderes ergibt. Sofern das betreffende Material nicht unter der genannten Creative Commons Lizenz steht und die betreffende Handlung nicht nach gesetzlichen Vorschriften erlaubt ist, ist für die oben aufgeführten Weiterverwendungen des Materials die Einwilligung des jeweiligen Rechteinhabers einzuholen.

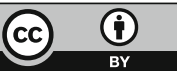

